\title{
Symbiotic Co-Evolution in Collaborative Human-Machine Decision Making: Exploration of a Multi-Year Design Science Research Project in the Air Cargo Industry
}

\author{
Daniel A. Döppner \\ Department of Information \\ Systems and Information \\ Management, \\ University of Cologne \\ doeppner@wim.uni-koeln.de
}

\author{
Patrick Derckx \\ Department of Information \\ Systems and Information \\ Management, \\ University of Cologne \\ derckx@wim.uni-koeln.de
}

\author{
Detlef Schoder \\ Department of Information \\ Systems and Information \\ Management, \\ University of Cologne \\ schoder@wim.uni-koeln.de
}

\begin{abstract}
The work world is set to undergo major changes thanks to advancements in automation and artificial intelligence and is beginning to promote new forms of collaboration. The transition from a technologysupporting environment to a collaborative environment in which people and technology work together to achieve their goals requires a fundamental change in the way we design, build, and ultimately deploy information systems.

Most work on information system design focuses on the effective augmentation of humans. However, little is known about constructing a sustainable mutually beneficial collaboration between human and machine. To better understand this relationship, we perform a case study drawing on ethnographic evidence collected during a multi-year design science research project with a major service provider for unit load device management in the air cargo industry that resulted in an artifact for human-machine collaboration (HMC). Our study takes a closer look at the co-evolution that emerges from the collaboration between human and artificial agents over time, in which both parties influence each other, the underlying tasks, and their environment. Our analysis reveals three facets of symbiotic co-evolution: agents' evolution, activity evolution, and structural evolution. The findings contribute to the HMC knowledge base and have implications for future HMC design initiatives.
\end{abstract}

\section{Introduction}

Artificial intelligence (AI) is becoming more capable of understanding and responding to situations and is thus having a significant impact on business (e.g., revenues, profits, operations, and employment). For the first time, machines can participate in both physical activities and intellectual tasks. Thus, technology is moving away from its augmenting and automating roles to become a powerful teammate that works with humans in various contexts. Recent examples of this transition include startup success prediction [14], object annotation [53], and software release planning [51]. As we incorporate smart technologies further into traditionally human processes, new forms of collaboration are emerging, making this topic exciting for both academics [55] and practitioners interested in exploring and exploiting this new form of teaming.

Shifting from the notion that AI should emulate or support humans, experts are beginning to predict that with the advancements in AI, business success will increasingly depend on people and machines collaborating with each other [57]. Thus, scholars suggest that we "consider AI not as the replacement of human beings, but as the empowerment and augmentation of humans" [20, 59]. This complementary perspective on the integration of machine and human intelligence suggests that technology design should focus on meaningful augmentation that complements humans instead of replacing them $[8,12]$.

This new form of collaboration will enable people and machines to engage in a symbiotic relationship, allowing them to do things that could not be done before and ultimately impacting our working environments. However, how this future of humanmachine collaboration (HMC) will look like depends on our design decisions.

Although the topic of human-machine symbiosis as the collaboration between human and technology has a long tradition [37, 58, 61, 67], current research is 
missing a conceptualization of co-evolutionary aspects. Reviewing the literature in the field of HMC and symbiotic systems reveals that most research focuses on the design of systems that aim to perform an activity and achieve a defined goal. Although the literature on HMC discusses learning and adaptation as a central facet, the relationship between interacting parties and the evolution of this relationship have not received much attention. This research gap impedes full theoretical understanding and systematic and purposeful managerial applications. Consequently, the objective of this study is to investigate the evolution of HMC and explore and synthesize patterns of symbiotic co-evolution.

Following a case study approach [68], we analyze data from a multi-year design science research (DSR) project that aimed to construct, evaluate, and introduce an IT artifact that incorporates the idea of humanmachine symbiosis in the air cargo industry.

Our study makes two contributions. First, we provide a conceptualization of symbiotic co-evolution by revealing three facets of co-evolution: agents' evolution, activity evolution, and structural evolution. Second, we provide rich description of coevolution in an HMC decision-making context. The findings are likely to be beneficial for practitioners who look for guidance in designing effective HMC.

The rest of this paper is organized as follows. In Section 2, we lay the foundation for this work by providing background knowledge on collaborative information systems and co-evolutionary theory. In Section 3, we describe our methodological approach and the research setting, and in Section 4, we present our findings. Finally, in Section 5, we discuss the results and outline implications and further research.

\section{Background}

\subsection{Human-Machine Collaboration}

Research on the design of collaboration between humans is ample and interdisciplinary, including work from psychology, organizational science, management science, and information systems. While the definition of collaboration differs across various disciplines, the following definition captures the main elements of the concept: "a coordinated, synchronous activity that is the result of a continued attempt to construct and maintain a shared conception of a problem" [50, p. 70]. Thus, collaborative problem solving is the mutual engagement of team members in a coordinated effort to solve a problem together. This definition is rooted in the collaboration research literature and is used in various papers $[3,4,26,61,67]$.
From an information systems perspective, the literature assigns collaborative information systems (CIS) to two roles: (1) CIS as a platform that supports collaborative activities in an organization among human collaborators and (2) CIS as a teammate in collaborative activities, for example through the application of AI. The latter is the focus of our study.

Most of the literature in this field can be divided into two clusters. First-generation CIS are considered information systems that support collaborative activities between human actors for tasks like product design [e.g., 54], data management [e.g., 48], and disaster management [e.g., 36, 60] and information systems that support the improvement of collaborative behavior [e.g., 5]. With the rise of smart machines, a new second generation of CIS began to emerge. These new systems are themselves collaborating actors, and the construction of these systems is focused on effective collaboration between human actors and artificial machine actors. There is ample research on information systems that exhibit such capabilities, which have been given various labels, including human-system collaboration, user-system collaboration, and human-agent teamwork [10]. For our research context, a particularly interesting term for this kind of system uses the metaphor of symbiosis [37]. The term symbiosis was coined in 1879 by the German plant pathology Heinrich Anton de Bary as "the living together of unlike organisms" that could lead to a co-evolutionary relationship.

\subsection{Co-Evolutionary Theory}

The term co-evolution was introduced back in the 1960s by the American population biologist Paul Ralph Ehrlich and the botanist Peter Hamilton Raven. Coevolution refers to the simultaneous evolutionary changes that occur within interacting populations [19, $21]$ and thus describes the reciprocal effects of two or more organisms on each other's evolution. In contrast to evolutionary theories, which view organizations, populations, or other entities in isolation, the coevolutionary perspective emphasizes the coupled evolution of multiple populations or forms and their embeddedness. These co-evolutions are likely to happen when different species have close ecological interactions with one another. Such close living can take different forms, ranging from being beneficial for all organisms involved (mutualism) to being harmful for one or more organisms (parasitism).

Interest in co-evolution grew, and as the concept spread quickly, it found application in other disciplines besides the natural sciences, including management science [34], the social sciences [42], and information systems [35]. In the latter, co-evolutionary theory has 
been applied in different research studies. The majority of studies use this theoretical perspective to examine alignment between information systems and organizations [2, 7, 65], their strategies [46], and their cultures or the relationship between information system personnel and information technology [44]. Other contexts include digital ecosystems [64], platforms [62], and IS development [41].

From an HMC perspective, the co-evolutionary perspective is a useful lens for considering the adaptive relationship between humans and machines in collaborative working constellations. Although, the coevolution of technology and humans has important implications [33], research on this theme is sparse. Our review on co-evolution in HMC shows that coevolution is mainly used to inform and discuss artifact design [27, 66] or provide a label for human-machine interaction $[11,28]$. A few studies use the concept to examine ongoing co-evolution, for example between a worker's task and a technology artifact [13].

Furthermore, we identified several shortcomings in the extant literature on CIS (second generation). First, prior studies on the design of collaborative information systems focus on developing system features that address cognitive alignment, interaction, and knowledge transfer between human and machine agents [e.g., 4, 67]. These studies focus on systems that are not primarily designed for collaboration, for instance traditional enterprise resource planning systems. Second, most empirical studies on CIS evaluate artifacts at single points in time. While most extant design research is on effective collaboration in terms of task completion and efficiency, to the best of our knowledge, no studies take a co-evolutionary perspective to examine the evolving relationship between humans and machines in collaborative settings, nor does existing research provide a better understanding of how this relationship evolves over time and might be guided. By examining an HMC in a real-world business context, this study addresses these shortcomings.

\section{Research Methodology}

Addressing the shortcomings of the literature, we performed a longitudinal case study analysis to explore the evolution that might take place in HMC settings and provide detailed descriptions of the phenomenon. Case studies are commonly used to increase knowledge about individuals, groups, or organizations and about social, political, and related phenomena [52]. HMC involves the interactions between individuals, groups, or organizations and information technology (IT) artifacts and presents a suitable domain for case study research. Furthermore, case study research is well suited for examining a particular phenomenon in its natural context [52] as it is the given case. According to Robson and McCartan's classification [49], our study's purpose can be classified as exploratory because it reveals what is happening, seeks new insights, and generates ideas and as descriptive because it illustrates a situation or phenomenon. In the next sections, we describe our research setting, our datacollection activities, and our analysis approach in more detail.

\subsection{Brief Case Description}

The focus of our research is HMC in complex decision-making contexts. The selected case is a DSR project started in 2012. The case company is a service provider for outsourced unit load device (ULD) management in the air cargo industry. ULDs are containers and pallets used for bundling and transporting freight, such as cargo, mail, or baggage in the air cargo industry.

The case company's main value proposition is to ensure ULD supply for its customers. It is responsible for a fleet of more than 100,000 ULDs for multiple airlines, which consists of 96 ULD types (different in shape, weight, and purpose) at more than 500 airports worldwide. To achieve this, the case company employs ULD dispatchers who continuously monitor and control the company's logistic network and reallocate the ULD stocks. This problem is called the empty ULD repositioning (EUR) problem [15].

At the time of writing, the artifact is used by a team of ULD dispatchers for their daily operations. The joint activity in which human and artificial agents work together entails relocating empty containers and pallets within the air cargo network to fulfill airlines' demands.

Before the artifact was introduced, decision making (i.e., data acquisition, information processing, information assessment, and identification and evaluation of movement options) was done manually. With the artifact, however, parts of this decisionmaking process can be done by a machine. This setup augments human's decision-making capabilities and frees up their cognitive resources for further valuecreating tasks.

Artifact development has passed through three iterations and has produced multiple knowledge moments. The first iteration focused on developing an intelligent decision-support system using rule-based expert system technology to prove that the complex decision making of EUR can be supported by IT [15, 18]. In the second iteration, the focus shifted to dividing labor between human and machine 
components and identifying a purposeful level of task automation [17]. The third iteration focused on an emerging co-evolution that became apparent when the ULD dispatchers interacted with the artifact [16]. This study is positioned after the third iteration and is based on our learnings and reflections from the previous iterations. The author team was engaged in all iterations of the project and have documented the evolution of the socio-technical artifact for the last five-and-a-half years.

The selected case suits our research objectives well for three main reasons: First, it fulfills the requirement for co-evolutionary studies necessitating data sets that span longer time periods [46] to observe changes that evolve over time. Second, the IT artifact is an instantiation of HMC. Finally, DSR projects involving close cooperation with problem-facing organizations enable the collection of rich data during artifact development and introduction.

\subsection{Data Collection}

Using elements of ethnographic research, we aimed to capture the collaboration between human agents and artificial agents and its evolution over time. More concretely, our methodological approach is inspired by design ethnography, a new form of engaged scholarship that bridges the gap between ethnographic research and DSR. While ethnographic studies provide a means to establish what is happening in practice and provide "IS researchers with rich insights into the human, the social, and organizational aspects of information systems" [43, p. 2], in design ethnography, the researcher goes beyond (participant) observation and actively engages with people in the field [6]. Ethnography was formerly used in IS research to study the changing nature of work and practice in relationship to information systems [6]. The "ethnographic approach that does not remove actors, actions and artifacts and that accounts for what happens in practice, provides a basis for building managerial implications that can serve managers as templates for reflection" [45, p. 3]. Thus, ethnography aims to gain a deeper understanding of the problem, the solution, and the environment [25, 43]. Zuboff, for example, conducted an in-depth ethnographic study that involved immersing herself in a company's workplace to uncover employees' IT usage [69].

Bridging research and practice [cf. rigor and relevance cycle in 29], the goal of the DSR paradigm is to extend human and organizational boundaries and capabilities by designing novel artifacts [24, 30]. To achieve this, DSR focuses on problem solving through the construction and evaluation of artifacts designed to solve real-world problems by enabling a transformation of an actual state into a desired state $[30,40]$. To achieve a solution that addresses the problem at hand, DSR opts for an iterative approach [29]. Each iteration generates a better understanding of (1) the given problem and its more abstract problem class, (2) possible solution features, more general design principles and design theories, and (3) on its scientific evaluation.

We triangulated our data to ensure they were sound and to increase the accuracy of our results. Triangulation provides a broader representation of the research object under study. We undertook two types of triangulation: data triangulation with varying types of data sources and points in time and methodological triangulation, which combines qualitative and quantitative data-collection methods. We collected qualitative data through participant observations, focus groups, expert interviews, and the analysis of archival materials and project documentation (e.g., meeting minutes of design workshops and telephone conferences, presentations, informal meetings, emails). The collected data cover a five-and-a-half-year period, starting with the initial project kick-off, deployment of the artifact, and roll-out and usage. We were highly involved in all phases of the project.

We used qualitative data-collection methods mainly to capture the opinions and impressions of the ULD dispatchers (human agents) and combined them with quantitative data capturing the machine's progress.

Our data collection incorporated different users collaborating with the same artifact to solve the same decision problem. This approach enables us to compare novice ULD dispatchers with experts who have multiple years of experience. Table 1 provides an overview of the collected data.

\section{Data Sources and Description}

Participant observations: Participant observation is a popular data-collection method in case study research and provides an effective means to collect ethnographic data. It involves systematically capturing actions performed by subjects. These observations served two purposes in the project at hand. On the one hand, we needed to understand users' work to derive a decision-making process model. On the other hand, we needed to capture the as-is state of case company's decision making to identify differences after the introduction and rollout of the artifact. Thus, we conducted participant observations at varying times during the project.

The latest observations took place in Apr 2017 and Dec 2017. Users with different levels of professional experience and experience using the artifact were observed in their work. If possible, we observed the same employees as at the beginning of the project, but this was not always possible due to employee turnover. Participant observations were conducted by two to three observers who took notes and audio-recorded the sessions. 
Focus groups: Collecting data from focus groups involves assembling small groups of peers to discuss particular topics. The interactive nature of these group discussions encourages participants to react to comments made by other participants. We conducted focus groups to discuss the artifact with ULD dispatchers. For example, we held a focus group to review new functionalities to solve concrete decision problems. These discussions were documented in notes.

Interviews: Interviews allow researchers to gain in-depth knowledge about a particular topic and collect opinions and impressions about the object under study. We mainly used interviews for evaluation purposes to examine the impact of the artifact on decision making. We conducted semistructured interviews with different ULD dispatchers who already used or began to use the artifact. Interviews were audio-recorded with the participants' consent and later transcribed.

Archival data: The DSR project was set up as a highly interactive and cooperative activity. Within the design team, we conducted design workshops and telephone conferences on a monthly basis. To date, 47 workshops have been held. The results were documented in protocols. These protocols not only contain design decisions but also reflect discussions. The protocols are particularly important because they capture project side effects and artifacts that directly or indirectly affect the ULD business.

Furthermore, we received informal feedback through email communication and discussions after and during system demonstrations. In addition, we archived the evolution of the program code to replay which and when artifact features were introduced.

Metrics: From a quantitative perspective, we collected data about system usage, decision making, and HMC's performance.

Typical questions included "How often was the system used and for what problems," "Who made a decision (human or machine)," "How many decisions were made," and "Was the decision informed by the machine or made by the human alone?"

Furthermore, we calculated measures to assess the system's quality by capturing the changes of the expert system's rule base and generated recommendations. We calculated the so-called hit rate, which gives information on the extent to which the artifact comes to the same decisions as human experts. These data contain information about whether the system makes the same decisions as users, whether users' decisions are informed by the system, and whether users (who were informed or not informed by the system) agree with the recommendations made by the system.

All metrics were collected over a longer time span, which allows us to inspect changes over time and compare system behavior before and after making it available to users.

\section{Table 1. Overview of fieldwork.}

\subsection{Data Analysis}

To explore our data and structure our findings, we align our research in the socio-technical system (STS) perspective. This perspective concludes that the social and technical aspects of information system phenomena are intertwined and that the boundaries between the two are not always clear [9, 39]. Thus, it conceptualizes information systems as two interrelated sub-systems: the technical system and the social system. The technical system consists of the tasks and technologies needed to produce products or services. The social system consists of the people and structure that comprise the relationship between them. STS is embedded in and influenced by an external environment. Furthermore, prior IS research argues that changes in STS are the result of misalignments within the STS - that is, among the socio-technical components [39], and that after the introduction of artifacts (technology), the interconnected sub-systems of the STS (people, tasks, structure) evolve with the artifact $[9,63]$.

\begin{tabular}{ll}
\hline STS Concepts & HMC \\
\hline People & Human Agents \\
Technology & Artificial Agents \\
Structure & Relationship \\
Task & Activity \\
\hline Table 2. Mapping human-machine \\
$\begin{array}{l}\text { collaboration to the socio-technical systems } \\
\text { perspective. }\end{array}$
\end{tabular}

In this study we take the same stance using STS as a framework to identify and organize the social and technical parts of information systems [9, 55]. Although the STS framework fits our research objective perfectly because it seems suitable for describing the evolution and change of information systems [39], we needed to make it applicable for the domain of HMC. Thus, we adopted the terminology described in Table 2.

For data analysis, we were inspired by techniques from the grounded theory methodology [22] and set up a five-step process. In Step 1, data from all data sources collected during the DSR project (see Section 3.2.) were merged using a central repository for all relevant media files. In Step 2, we started with initial coding, analyzing the collected data to identify and formulate ideas, themes, and issues relevant for this study. We carefully screened the underlying data to identify evolution in the STS components and took notes that we later discussed and consolidated. This resulted in a set of nine first-order themes related to evolutionary behavior. When creating the first-order themes, we took care to map the data sources to the 
themes so that no information was lost. We also wanted to check which data sources provide information on the same first-order themes. These links are shown in Figure 1. In Step 3, we performed focus coding, aggregating identified themes into higher-order themes. We ended up with a set of seven second-order themes. Then, in Step 4, we conducted axial coding and classified the findings into three facets of coevolution. Finally, in Step 5, we are disseminating the results in the context of this publication.

\section{Findings}

Our data analysis revealed three facets of coevolution: agents' evolution, activity evolution, and structural evolution (see Figure 1). For each theme, we present a brief description and provide concrete examples from the EUR case that demonstrate how the evolution manifested and make the themes more tangible for practitioners. Figure 1 shows the results of the five-step analysis process and indicates the link between our data sources and themes. For example, the machine's evolution is captured in the metrics data and was revealed in our analysis of the hit rate over time.

\subsection{Agents' Evolution}

For HMC, both sides of the collaboration - the machine and the human (the user) — can be seen as agents of the system. Our investigations of the available data from the metrics and interviews show that both agents have evolved. The data confirm that the artifact has become better over time by developing and proposing solutions that are more similar to those of human experts. It is evident that the artifact's knowledge gaps have been continuously filled and that its information-processing capabilities have improved over time.
Machine's evolution. The accuracy of the recommendations made by the machine increased with use of the system. This increased accuracy can be seen by examining the hit rate. The hit rate relates the machine's recommendations to the actual actions performed by people. The hit rate changed due to the expansion of the machine's rule base, which is used to derive recommendations. Furthermore, the users mentioned they noticed an increase in the machine's solution capacities, i.e. the quality of recommendations.

Human's evolution. Whether workers perceived a change or not, their work changed with use of the machine. For example, the machine explains the reasoning for suggested solutions and can support training. Thus, a novice ULD dispatcher can be partially trained by the machine and can avoid depending on an experienced colleague for permanent support. The system takes on the role of the trainer, playing a decisive role in the development of new ULD dispatchers. An ULD dispatcher noted that an experienced dispatcher already knows about $90 \%$ of the recommendations and probably learns less than a novice who can be fully supported by the decision support system (DSS). We observed that experienced ULD dispatchers mentioned that they "overlook complex [repositioning alternatives] with multi-sector flights but the DSS gives [them] suggestion(s)." Thus, they were also able to learn from the machine. In complex decision situations, in particular, the system pointed out new ways to solve problems. They also mentioned that they use the system to confirm their decisions by requesting recommendations. Thus, the explanation component of the system enabled them to better evaluate decisions and "prevent a bad decision." In sum, the evolution of the human was evident in various factors (e.g., experience level, self-confidence, and susceptibility to failure).

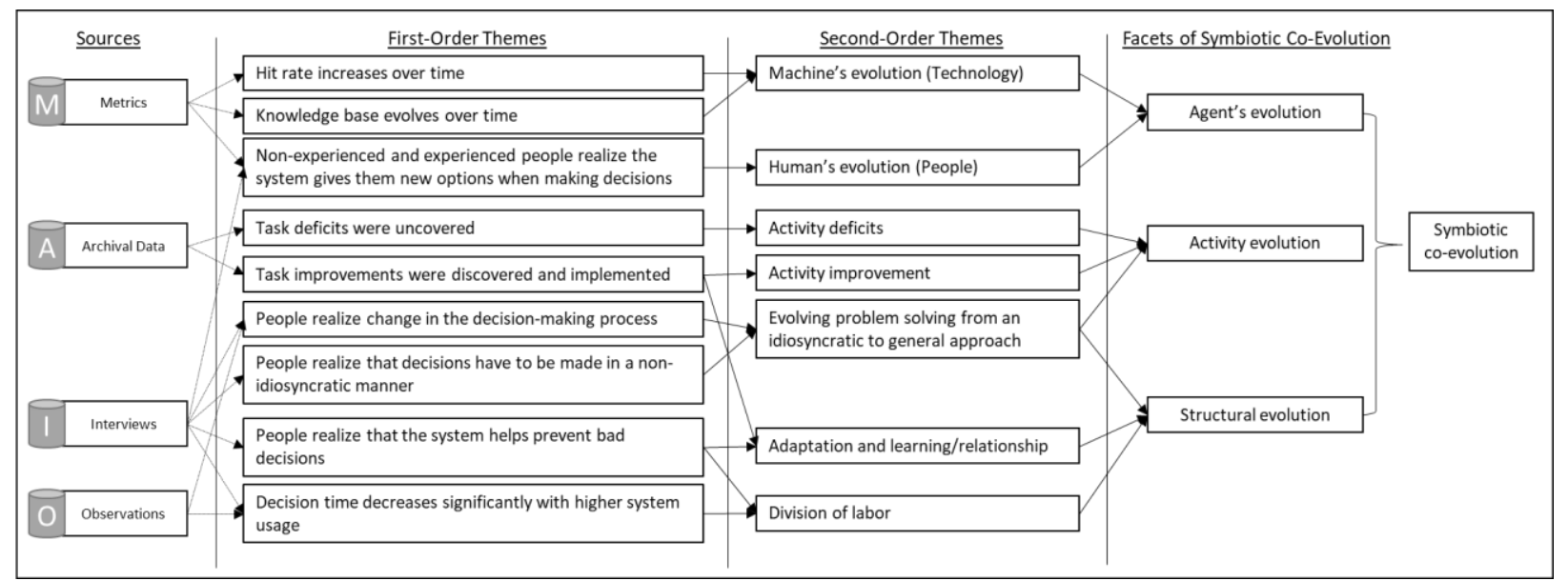

Fiqure 1. Data structure. 


\subsection{Activity Evolution}

Our analysis showed that even the tasks themselves are not invariable elements in an HMC. In addition to the development of the agents, the activities themselves developed further throughout the project.

Activity deficits. The introduction of the system not only enabled the simultaneous consideration of larger data volumes and a global view of the ULD network but has also led ULD dispatchers to question their current decision-making processes.

After more intensive work with the system, the ULD dispatchers discovered that the processes had to be changed and questioned the current process steps (e.g., manual labor-intensive data acquisition and preprocessing). They noticed that the system "makes [their] work more automatic [so they] skip some [information] processing [steps]."

Activity improvement. A key result of the DSR project is that effective control of the network by the machine is highly dependent on accurate facts. Thus, derived from the design workshops, two new human roles were created that are closely connected to the machine's effectiveness and thus the outcome of the collaboration. One new role was established that is responsible for the evolution of the system's knowledge base. Another new role was created to continuously monitor and adjust inventory safety stock levels [38].

Generalizing idiosyncratic problem-solving approaches. There was a noticeable change in how decisions are made. Through observations of the ULD dispatchers, we were able to document a partly personindividual unconventional decision-making behavior. After the system was introduced and used, processes have been partly standardized or have been actively moved in this direction by the users. For example, during the project, we often heard "It has always been done this way" and "This is a peculiarity of the airline." We transformed these sentiments into more general problem-solving knowledge that is applicable to a broader range of problems and synthesizes different problem-solving approaches.

\subsection{Structural Evolution}

In addition to the evolutionary patterns of agents and activities, our data analysis showed that the collaboration setup is also subject to evolution. This structural development is characterized mainly by the changing division of labor between the two actors and its dynamic function allocation due to mutual adaptation and learning.
Division of labor. Before introducing the HMC, ULD dispatchers had to manually collect all relevant information before planning. Although this had already been done with IT support before, the existing systems were not able to prepare the information for decisions but reacted much more to ad hoc requests from users. An analysis of historical ULD repositioning decisions revealed that with the introduction of the HMC, work was redistributed such that the machine can take on cognitively more demanding tasks and be available to the ULD dispatchers as a kind of teammate. Furthermore, interviews with the ULD dispatchers confirmed that they perceived the system to help them avoid wrong decisions as it continuously collects the necessary data itself and informs them early on.

In addition, they mentioned that the perceived decision time was also shortened by the constant presence of the machine. The reduced decision time allowed the ULD dispatchers to improve their analytical skills, which in turn had an impact on the quality of their decisions.

Adaptation and learning. As other authors have already mentioned [67], HMC is not a one-time event but a new way of using and perceiving machines. In the context of this new relationship, intensive cooperation leads to mutual learning and adaptation among collaborators. The ULD dispatchers mentioned, for example, that the machine proposed alternatives that were not normally known to them, or that the feasibility of these alternatives was difficult to verify due to time constraints. The machine agent now conducts these activities automatically and communicates to the ULD dispatchers. As one ULD dispatcher explained to us, "with DSS you can see the whole [air cargo network]."

On the other hand, applying machine learning techniques, the machine can derive new knowledge from past decisions and adapt to user behavior. For example, the machine incorporates association rulemining features to reveal missing rules from historical decisions that are then used to extend the rule base.

\section{Discussion and Conclusion}

Taking a STS perspective on HMC $[58,61,67]$ in a complex decision-making context, we set out to explore the symbiotic relationship between human and technology. We found that there are three facets of symbiotic co-evolution: agents' evolution, activity evolution, and structural evolution (see Figure 2).

This study provides the first empirical insights into the phenomenon of symbiotic co-evolution in the context of collaborative human-machine decision making over an extended time span. The results show 
that a kind of evolution takes place within the collaborative human-machine decision-making setting. Since available knowledge about this phenomenon is scarce, the implications of this study are relevant for both theory and practice.

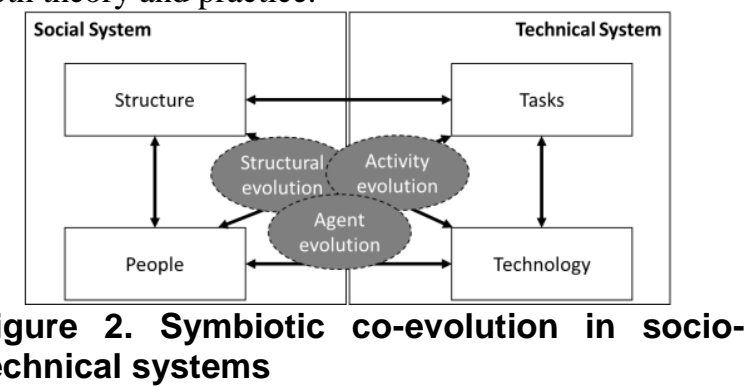

\subsection{Theoretical Implications}

Answering calls for studies in the field of humanmachine teaming [56], we contribute to the design knowledge base with our conceptualization of the symbiotic co-evolution that might occur in HMC settings and provide a framework to describe facets of this symbiotic co-evolution.

This study is exploratory in nature instead of having a confirmatory focus, so we do not consider the facets conclusive but as a starting point. While traditional DSR details the construction and evaluation of IT artifacts by the research community, the case at hand represents the construction of a complex IT artifact developed in a real-world practical context [31]. Therefore, making rich descriptions of such cases available contributes additional data sources for future research projects. The findings of this paper can be classified as an attempt at "generalizing from data to description" [32] and aims to contribute toward a theory for analysis and description [23].

The findings demonstrate that symbiotic coevolution is a useful research lens to describe change and adaptation in STS and extends prior work in this direction [e.g., 39] by conceptualizing technology as collaborating agents.

Furthermore, our findings extend the existing knowledge on HMC design, which focuses more on goal achievement and effective collaboration in activities [e.g., 58, 61, 67] than on the mutual development of the collaborating parties and their relationship. In this sense, our results might stimulate discussion on new problem classes focusing on the purposeful design of symbiotic co-evolution. Consequently, our findings inform the IS design knowledge that guides the construction of humanmachine symbiotic artifacts in other contexts.

\subsection{Practical Implications}

The rapid advances of smart machines have triggered a changing perspective in system development from solving problems by introducing specific rather than static artifacts to constructing technologies that are designed to evolve over time through interaction with humans either autonomously or with minimal involvement of human designers.

The capabilities of such IT artifacts both enable and constrain managers and consumers as they implement and redesign work systems and the allocation of human resources [1]. We argue that collaboration design between humans and machines is inevitable. The development of such collaboration may be time consuming and resource intensive, so managers need to acknowledge the existence of this dynamic collaborative relationship for future design initiatives, not only in terms of pure technological artifact design but also in terms of the organizational design technology is woven into.

Besides the effects on the design of new forms of collaborative information systems, our results could also influence the design of future (digital) organizations currently on the path to digital innovation and transformation. Thus, organizational design should consider that with the emergence of smart machines, human-machine collaboration is becoming inevitable and must be considered when designing workplaces and training.

\subsection{Limitations and Future Research}

As with every research, our study does not come without limitations. Although a single case study design affords the opportunity to generalize observations and measurements [32] and while the selected case serves as a perfect exemplary instantiation, the findings may not be generalizable. As such, further research is needed to replicate our findings across different contexts.

Moreover, while we examined collaboration in a monitor-and-control decision setting, other scenarios might be interesting as well. Besides classical decisionsupport topics, process-driven enterprise resource systems [67] and contexts with a higher degree of social interaction (e.g., human resource management and customer relationship management) also provide interesting contexts.

Furthermore, as other authors have mentioned, evolution might be planned or unplanned as it is difficult to derive sound rules to trigger co-evolution [47]. Therefore, it would be interesting to analyze HMC at different points in time, examine the environmental state, and identify events that trigger 
evolutionary change. Nevertheless, we hope our findings stimulate an interesting discussion of $\mathrm{HMC}$ and IS design in general.

\section{References}

[1] Alter, S., "18 Reasons Why IT-Reliant Work Systems Should Replace 'The IT Artifact' as the Core Subject Matter of the IS Field", Communications of the Association for Information Systems 12(1), 2003.

[2] Amarilli, F., M. van Vliet, and B.V. den Hooff, "An Explanatory Study on the Co-evolutionary Mechanisms of Business IT Alignment", ICIS 2017 Proceedings, (2017).

[3] Babaian, T., W. Lucas, and H. Topi, "Improving ERP Usability Through User-System Collaboration", International Journal of Enterprise Information Systems 2(3), 2006, pp. 10-23.

[4] Babaian, T., W. Lucas, J. Xu, and H. Topi, "Usability through System-User Collaboration”, DESRIST 2010, Springer Berlin Heidelberg (2010), 394-409.

[5] Barthe-Delanoë, A.-M., A. Montarnal, S. Truptil, F. Bénaben, and H. Pingaud, "Towards the agility of collaborative workflows through an event driven approach Application to crisis management", International Journal of Disaster Risk Reduction, 2018.

[6] Baskerville, R.L., and M.D. Myers, "Design ethnography in information systems: Design ethnography", Information Systems Journal 25(1), 2015, pp. 23-46.

[7] Benbya, H., and B. McKelvey, "Using coevolutionary and complexity theories to improve IS alignment: a multilevel approach", Journal of Information technology 21(4), 2006, pp. 284-298.

[8] Bichler, M., U. Frank, D. Avison, et al., "Theories in Business and Information Systems Engineering", Business \& Information Systems Engineering 58(4), 2016, pp. 291-319.

[9] Bostrom, R.P., and J.S. Heinen, "MIS Problems and Failures: A Socio-Technical Perspective PART I: THE CAUSES", MIS Quarterly 1(3), 1977, pp. 17-32.

[10] Bradshaw, J.M., M. Sierhuis, A. Acquisti, et al., "Adjustable Autonomy and Human-Agent Teamwork in Practice: An Interim Report on Space Applications", In H. Hexmoor, C. Castelfranchi and R. Falcone, eds., Agent Autonomy. Springer US, 2003, 243-280.

[11] Brangier, É., and S. Hammes-Adelé, "Beyond the Technology Acceptance Model: Elements to Validate the Human-Technology Symbiosis Model", In Ergonomics and Health Aspects of Work with Computers. Springer, Berlin, Heidelberg, 2011, 13-21.

[12] Brynjolfsson, E., and A. McAfee, The Second Machine Age: Work, Progress, and Prosperity in a Time of Brilliant Technologies, W. W. Norton \& Company, 2014.

[13] Bunch, L., J.M. Bradshaw, M. Carvalho, et al., "HumanAgent Teamwork in Cyber Operations: Supporting Coevolution of Tasks and Artifacts with Luna”, In Multiagent System Technologies. Springer, Berlin, Heidelberg, 2012, 53-67.

[14] Dellermann, D., N. Lipusch, P. Ebel, K.M. Popp, and J.M. Leimeister, "Finding the Unicorn: Predicting Early Stage Startup Success through a Hybrid Intelligence Method", (2017).
[15] Döppner, D.A., P. Derckx, and D. Schoder, "An Intelligent Decision Support System for the Empty Unit Load Device Repositioning Problem in Air Cargo Industry", Proceedings of the 51st Hawaii International Conference on System Sciences (HICSS 2018), (2018).

[16] Döppner, D.A., P. Derckx, and D. Schoder, "IDSS4CEUR: An Intelligent Decision Support System for Collaborative Empty Unit Load Device Repositioning", DESRIST 2018, (2018).

[17] Döppner, D.A., R.W. Gregory, D. Schoder, and H. Siejka, "Exploring Design Principles for Human-Machine Symbiosis: Insights from Constructing an Air Transportation Logistics Artifact", ICIS 2016 Proceedings, (2016).

[18] Döppner, D.A., D. Schoder, and H. Siejka, "Big Data and the Data Value Chain: Translating Insights from Business Analytics into Actionable Results - The Case of Unit Load Device (ULD) Management in the Air Cargo Industry”, ECIS 2015 Research-in-Progress Papers, (2015). [19] Ehrlich, P.R., and P.H. Raven, "Butterflies and Plants: A Study in Coevolution", Evolution 18(4), 1964, pp. 586-608.

[20] Fischer, G., "Beyond 'couch potatoes': from consumers to designers", Proceedings. 3rd Asia Pacific Computer Human Interaction (Cat. No.98EX110), (1998), 2-9.

[21] Futuyma, D.J., and M. Slatkin, Coevolution, Sinauer Associates Inc.,U.S., Sunderland, Mass, 1983.

[22] Glaser, B.G., and A.L. Strauss, The discovery of grounded theory: Strategies for qualitative research, Transaction publishers, 2009.

[23] Gregor, S., "The Nature of Theory in Information Systems", MIS Quarterly 30(3), 2006, pp. 611-642.

[24] Gregor, S., and A.R. Hevner, "Positioning and Presenting Design Science Research for Maximum Impact", MIS Quarterly 37(2), 2013, pp. 337-356.

[25] Gregory, R.W., and J. Muntermann, "Research NoteHeuristic Theorizing: Proactively Generating Design Theories", Information Systems Research 25(3), 2014, pp. 639-653.

[26] Grosz, B.J., and S. Kraus, "Collaborative plans for complex group action”, Artificial Intelligence 86(2), 1996, pp. 269-357.

[27] Guyet, T., C. Garbay, and M. Dojat, "Knowledge construction from time series data using a collaborative exploration system", Journal of Biomedical Informatics 40(6), 2007, pp. 672-687.

[28] Hadorn, B., M. Courant, and B. Hirsbrunner, "Holistic integration of enactive entities into cyber physical systems", 2015 IEEE 2nd International Conference on Cybernetics (CYBCONF), (2015), 281-286.

[29] Hevner, A.R., "A three cycle view of design science research", Scandinavian journal of information systems 19(2), 2007, pp. 1-6.

[30] Hevner, A.R., S.T. March, J. Park, and S. Ram, "Design Science in Information Systems Research", MIS Quarterly 28(1), 2004, pp. 75-105.

[31] Iivari, J., "A paradigmatic analysis of information systems as a design science", Scandinavian Journal of Information Systems 19(2), 2007, pp. 5.

[32] Lee, A.S., and R.L. Baskerville, "Generalizing Generalizability in Information Systems Research", Information Systems Research 14(3), 2003, pp. 221-243. 
[33] Lee, J.D., "Review of a Pivotal Human Factors Article: 'Humans and Automation: Use, Misuse, Disuse, Abuse", Human Factors 50(3), 2008, pp. 404-410.

[34] Lewin, A.Y., and H.W. Volberda, "Prolegomena on Coevolution: A Framework for Research on Strategy and New Organizational Forms", Organization Science 10(5), 1999, pp. 519-534.

[35] Lewis, G., "Concepts from Coevolution in Information Systems", AMCIS 2008 Proceedings, 2008.

[36] Li, J., Q. Li, C. Liu, S. Ullah Khan, and N. Ghani, "Community-based collaborative information system for emergency management", Computers \& Operations Research 42, 2014, pp. 116-124.

[37] Licklider, J.C.R., "Man-Computer Symbiosis", IRE Transactions on Human Factors in Electronics HFE-1(1), 1960, pp. 4-11.

[38] Lu, H.-A., and C.-Y. Chen, "Safety stock estimation of unit load devices for international airline operations", Journal of Marine Science and Technology 20(4), 2012, pp. 431-440.

[39] Lyytinen, K., and M. Newman, "Explaining information systems change: a punctuated socio-technical change model", European Journal of Information Systems 17(6), 2008, pp. 589-613.

[40] March, S.T., and G.F. Smith, "Design and natural science research on information technology", Decision Support Systems 15(4), 1995, pp. 251-266.

[41] McKelvey, B., and H. Benbya, "Toward a complexity theory of information systems development", Information Technology \& People 19(1), 2006, pp. 12-34.

[42] Mitleton-Kelly, E., and L.K. Davy, "The Concept of 'Co-evolution' and Its Application in the Social Sciences: A Review of the Literature", In Co-evolution of Intelligent Socio-technical Systems. Springer, Berlin, Heidelberg, 2013, 43-57.

[43] Myers, M., "Investigating Information Systems with Ethnographic Research", Commun. AIS 2(4es), 1999.

[44] Niederman, F., T.W. Ferratt, and E.M. Trauth, "On the Co-Evolution of Information Technology and Information Systems Personnel", SIGMIS Database 47(1), 2016, pp. 2950 .

[45] Niemimaa, E., "Crafting an Information Security Policy: Insights from an Ethnographic Study", ICIS 2016 Proceedings, 2016.

[46] Peppard, J., and K. Breu, "Beyond Alignment: A Coevolutionary View of the Information Systems Strategy Process", ICIS 2003 Proceedings, (2003).

[47] Ramage, M., "Forms and processes of information systems evolution", (2006).

[48] Reschenhofer, T., M. Bhat, A. Hernandez-Mendez, and F. Matthes, "Lessons Learned in Aligning Data and Model Evolution in Collaborative Information Systems", Proceedings of the 38th International Conference on Software Engineering Companion, ACM (2016), 132-141.

[49] Robson, C., and K. McCartan, Real World Research, Wiley, Hoboken, 2015.

[50] Roschelle, J., and S.D. Teasley, "The Construction of Shared Knowledge in Collaborative Problem Solving", In C. O'Malley, ed., Computer Supported Collaborative Learning. Springer Berlin Heidelberg, Berlin, Heidelberg, 1995, 69-97.
[51] Ruhe, G., and A. Ngo The, "Hybrid Intelligence in Software Release Planning", International Journal of Hybrid Intelligent Systems 1(1-2), 2004, pp. 99-110.

[52] Runeson, P., and M. Höst, "Guidelines for conducting and reporting case study research in software engineering", Empirical Software Engineering 14(2), 2009, pp. 131.

[53] Russakovsky, O., L.J. Li, and L. Fei-Fei, "Best of both worlds: Human-machine collaboration for object annotation", 2015 IEEE Conference on Computer Vision and Pattern Recognition (CVPR), (2015), 2121-2131.

[54] Salama, S., F. Mnale, J. Park, A. Abdelhalim, and A. Eltawil, "Mobi-Manager: A Collaboration Platform for Production Management: the case of the Egyptian Furniture Industry", PACIS 2017 Proceedings, (2017).

[55] Sarker, S., S. Chatterjee, and X. Xiao, "How 'Sociotechnical' is our IS Research? An Assessment and Possible Ways Forward”, ICIS 2013 Proceedings, (2013).

[56] Seeber, I., E. Bittner, R.O. Briggs, et al., "Machines as Teammates: A Collaboration Research Agenda", Proceedings of the 51st Hawaii International Conference on System Sciences, (2018).

[57] Shook, E., and M. Knickrehm, Future Workforce: Reworking the Revolution, accenture Strategy, 2018.

[58] Silverman, B.G., "Human-Computer Collaboration", Human-Computer Interaction 7(2), 1992, pp. 165-196.

[59] Stefik, M., “The Next Knowledge Medium”, AI Mag. 7(1), 1986, pp. 34-46.

[60] Tahir, O., E. Andonoff, C. Hanachi, et al., "A Collaborative Information System Architecture for ProcessBased Crisis Management", Knowledge-Based Intelligent Information and Engineering Systems, Springer, Berlin, Heidelberg (2008), 630-641.

[61] Terveen, L.G., "Overview of human-computer collaboration", Knowledge-Based Systems 8(2), 1995, pp. $67-81$.

[62] Tiwana, A., B. Konsynski, and A.A. Bush, "Research Commentary-Platform Evolution: Coevolution of Platform Architecture, Governance, and Environmental Dynamics", Information Systems Research 21(4), 2010, pp. 675-687.

[63] Trist, E.L., The Evolution of Socio-technical Systems: A Conceptual Framework and an Action Research Program, Ontario Ministry of Labour, Ontario Quality of Working Life Centre, 1981.

[64] Um, S., and Y. Yoo, "The Co-Evolution of Digital Ecosystems", ICIS 2016 Proceedings, (2016).

[65] Vessey, I., and K. Ward, "The Dynamics of Sustainable IS Alignment: The Case for IS Adaptivity", Journal of the Association for Information Systems 14(6), 2013.

[66] Xia, C., and P. Maes, "The Design of Artifacts for Augmenting Intellect", Proceedings of the 4th Augmented Human International Conference, ACM (2013), 154-161.

[67] Xu, J., and H. Topi, "A Conceptual Model for Usersystem Collaboration: Enhancing Usability of Complex Information Systems", Communications of the Association for Information Systems 41(1), 2017, pp. 775-800.

[68] Yin, R.K., Case Study Research: Design and Methods, SAGE Publications, Inc, Thousand Oaks, Calif, 2003.

[69] Zuboff, S., In the age of the smart machine: The future of work and power, Basic Books, New York, NY, 1988. 